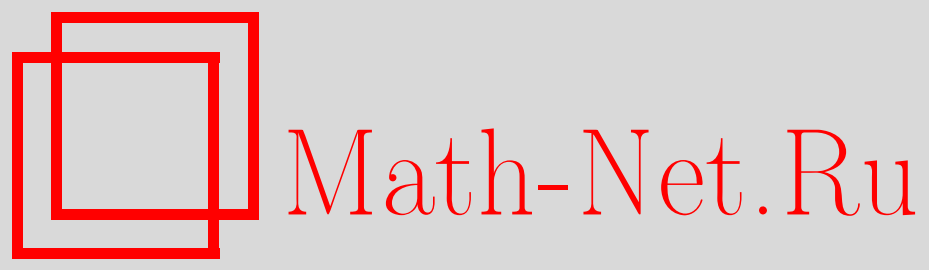

В. С. Рабинович, Алгебры сингулярных интегральных операторов на составных контурах с точками завихрения, Функи. анализ и его прил., 1996, том 30, выпуск 3, 85-87

DOI: https://doi.org/10.4213/faa541

Использование Общероссийского математического портала MathNet.Ru подразумевает, что вы прочитали и согласны с пользовательским соглашением

http://www . mathnet.ru/rus/agreement

Параметры загрузки:

IP: 18.208 .226 .222

26 апреля 2023 г., 17:56:08

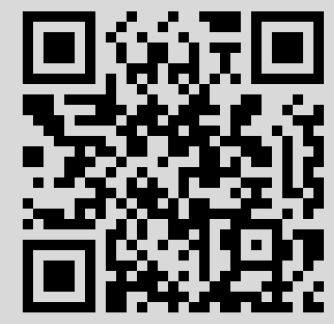




\title{
Алгебры сингулярных интегральных операторов на составных контурах с точками завихрения
}

\author{
(c) $1996 . \quad$ В. С. РАБинович ${ }^{1}$
}

Даны условия фредгольмовости и формула индекса для операторов из банаховой алгебры, порожденной сингулярными интегральными операторами (СИО) на составных контурах с точками завихрения и коэффициентами, имеющими ограниченные разрывы второго рода. Исследованию фредгольмовости различных классов сингулярных интегральных операторов с кусочно-непрерывными коэффициентами на кусочно-ляпуновских контурах посвящены монографии [1-3] и др. Алгебры, порожденные СИО с кусочно-непрерывными коэффициентами на замкнутых контурах с точками логарифмического завихрения, рассмотрены в [4]. Работы $[5,6]$ посвящены $C^{*}$-алгебрам, порожденным СИО на составных контурах с узлами, являющимися точками завихрения менее общего характера, чем здесь.

Если $B$ - банахово пространство, то через $\mathfrak{L}(B)$ обозначается банахова алгебра всех ограниченных операторов, действующих в $B$, а через $\mathfrak{K}(B)-$ двусторонний идеал компактных операторов в ней.

Через $L_{p, \beta}\left(\mathbb{R}_{+}, \mathbb{C}^{N}\right), p \in(1, \infty), \beta \in \mathbb{R}$, обозначим банахово пространство комплексных вектор-Функций $u(r)=\left(u_{1}(r), \ldots, u_{N}(r)\right)$ с нормой $\|u\|_{L_{p, \beta}}=$ $\left(\int_{\mathbb{R}_{+}} r^{p \beta} \sum_{j=1}^{N}\left|u_{j}(r)\right|^{p} d r\right)^{1 / p}$. Матрицу-функцию $a(\lambda) \in L_{\infty}\left(\mathbb{R}_{\beta+1 / p}\right) \otimes \mathfrak{L}\left(\mathbb{C}^{N}\right)$, где $\mathbb{R}_{\mu}=\{z \in \mathbb{C}: \operatorname{Im} z=\mu\}$, будем называть меллиновским мультипликатором в $L_{p, \beta}\left(\mathbb{R}_{+}, \mathbb{C}^{N}\right)$, если оператор меллиновской свертки $a(\mathscr{D})$ ограничен в $L_{p, \beta}\left(\mathbb{R}_{+}, \mathbb{C}^{N}\right)$. Через $\mathfrak{N}_{p, \beta}(N), p \in(1, \infty)$, будем обозначать банахову алгебру меллиновских мультипликаторов в $L_{p, \beta}\left(\mathbb{R}_{+}, \mathbb{C}^{N}\right)$ с нормой, индуцированной операторной нормой $a(\mathscr{D})$, а через $\mathscr{R}_{p, \beta}(N)$ - банахову алгебру ограниченных непрерывных на $\mathbb{R}_{+} N \times N$-матриц-функций $a(r, \cdot)$ со значениями в $\mathfrak{N}_{p, \beta}(N)$, нормой $\|a\|_{\mathscr{R}_{p, \beta}}=\sup _{r \in \mathbb{R}}\|a(r, \cdot)\|_{\mathfrak{N}_{p, \beta}(N)}$ и естественными операциями сложения и умножения матрищ-функций. Через $\mathfrak{J}_{p, \beta}^{0}(N)$ обозначим идеал в $\mathscr{R}_{p, \beta}(N)$, состоящий из таких матриц-функций $a \in \mathscr{R}_{p, \beta}(N)$, что $\lim _{r \rightarrow+0}\|a(r, \cdot)\|_{\mathfrak{N}_{p, \beta}(N)}$ $=0$, а через $\mathscr{R}_{p, \beta}^{+}(N)$ - факторалгебру $\mathscr{R}_{p, \beta}(N) / \mathfrak{J}_{p, \beta}^{0}(N)$ с естественной нормой.

Будем говорить, что простая (гомеоморфная сегменту) спрямляемая незамкнутая дуга $\gamma \subset \mathbb{C}$ с концами $x_{1}, x_{2}$ принадлежит классу $\mathfrak{R}$, если:

1) $\gamma \backslash\left\{x_{1}, x_{2}\right\}$ - локально ляпуновская дуга;

2) пусть $x-x_{j}=\varphi_{j}(t)=t \exp i \omega_{j}(t), t \in\left[0, t_{j}\right]$, где $\omega_{j}(t)$ - вещественная функция, параметризация дуги $\gamma$ в окрестности конца $x_{j}$. Будем предполагать, что $\omega_{j}(t)=\delta_{j} \ln t+\theta_{j}(t)$, где $\delta_{j} \in \mathbb{R}, \theta_{j}(t) \in C^{\infty}\left(\left(0, t_{j}\right]\right)$, и

$$
\lim _{t \rightarrow+0}(t \partial / \partial t)^{k} \theta_{j}(t)=0, \quad k \in \mathbb{N} .
$$

${ }^{1}$ Работа выполнена при поддержке Российского фонда фундаментальных исследований, грант №93-011-280. 
Примерами функций $\theta(t)$, удовлетворяющих условию (1), являются $|\ln t|^{\alpha}$ и $a+$ $b \sin |\ln t|^{\alpha}, a, b \in \mathbb{R}, \alpha \in(0,1)$.

Пусть $\Gamma$ - ориентированный контур в плоскости $\mathbb{C}$, состоящий из конечного числа дуг класса $\mathfrak{R}$. Предполагается, что любые две дуги могут пересекаться только в концевых точках, и пусть $F \subset \Gamma$ - множество концевых точек этих дуг. Точки множества $F$ будем называть узлами. Пусть $z \in F, N(z)$ - число дуг, составляющих узел $z$, и параметризации дуг $\gamma_{j}$, составляющих узел $z$, имеют следующий вид: $x-z=\varphi_{j}^{z}(t)=t \exp i \omega_{j}^{z}(t), t \in\left[0, t_{j}\right], j=1, \ldots, N(z)$, $\omega_{j}^{z}(t)=\delta_{z} \ln t+\theta_{j}^{z}(t), \delta_{z} \in \mathbb{R}$. Будем предполагать, что существует $t_{0}>0$, такое, что $\theta_{j}^{z}(t) \in\left(\alpha_{j}, \beta_{j}\right)$, когда $t \in\left(0, t_{0}\right]$, где $0 \leqslant \alpha_{1}<\beta_{1}<\alpha_{2}<\beta_{2}<$ $\cdots<\alpha_{N}<\beta_{N}<2 \pi$. Через $P S V(\Gamma, F)$ обозначим $C^{*}$-алгебру, полученную замыканием по sup-норме на $\Gamma$ множества ограниченных непрерывно дифференцируемых на $\Gamma \backslash F$ функций, таких, что $\lim _{\Gamma \ni x \rightarrow x_{j}}\left|x-x_{j}\right| a^{\prime}(x)=0$ для любого узла $x_{j}$. Функции класса $P S V(\Gamma, F)$ могут иметь ограниченные разрывы второго рода в окрестности узлов.

Пусть $L_{p}(\Gamma, \omega)$ - пространство функций, суммируемых с весом $\omega(x)=$ $\prod_{m=1}^{L}\left|x-x_{m}\right|^{\beta_{m}}, x_{m} \in F, \beta_{m} \in(-1 / p, 1-1 / p)$, и $p$-й степенью $(1<p<\infty)$, a $S_{\Gamma}$ - сингулярный интегральный оператор на контуре $\Gamma$, ограниченный в $L_{p}(\Gamma, \omega)$, так как дуги $\gamma_{j}$, составляющие контур, удовлетворяют условию Карлесона.

Определим локальные символы $\sigma^{x}(a)$ оператора умножения на функцию $a(x)$ $\in P S V(\Gamma, F)$. Пусть $x \in \Gamma \backslash F$; тогда $\sigma^{x}(a)=a(x)$. Если же $x \in F$, то локальный символ $\sigma^{x}(a)$ есть диагональная матрица $\operatorname{diag}\left(a_{1}^{x}(t), \ldots, a_{N}^{x}(t)\right)$, где $a_{k}^{x}(t)=\left.a\right|_{\gamma_{k}}\left(x+\varphi_{k}^{x}(t)\right), t \in\left(0, t_{0}\right], t_{0}$ - достаточно малое число.

Определим теперь локальные символы $\sigma^{x}\left(S_{\Gamma}\right)$. Пусть $x \in \Gamma \backslash F$; тогда $\sigma^{x}\left(S_{\Gamma}\right)$ $=\operatorname{sgn} \xi, \xi \in \mathbb{R}$. Если $x \in F$, то $\sigma^{x}\left(S_{\Gamma}\right)$ есть матрица-функция $\Omega^{x}(t, \lambda)=$ $\left(\Omega_{j k}^{x}(t, \lambda)\right)_{j k=1}^{N(x)} \in \mathscr{R}_{p, \beta}(N)$, где $\Omega_{j k}^{x}(t, \lambda)$ вычисляются по формулам: $\Omega_{j k}^{x}(t, \lambda)=$ $\pm \exp \left[\lambda\left(\theta_{k}^{x}(t)-\theta_{j}^{x}(t)-\pi\right)\right](\operatorname{sh} \pi \lambda)^{-1}, k>j, \Omega_{j k}^{x}(t, \lambda)= \pm \exp \left[\lambda\left(\theta_{k}^{x}(t)-\theta_{j}^{x}(t)+\right.\right.$ $\pi)](\operatorname{sh} \pi \lambda)^{-1}, k<j, \Omega_{j j}^{x}(\lambda)= \pm \operatorname{cth}(\pi \lambda)$. Здесь $t \in(0, s]$, где $s$ достаточно мало, знак + берется, если контур $\Gamma$ ориентирован так, что кривая $\gamma_{k}$ выходит из узла, и знак - берется, если кривая $\gamma_{k}$ входит в узел, $\lambda \in\{\zeta=\mu+i \nu \in \mathbb{C}$ : $\left.\nu=-\delta_{x} \mu+\beta_{x}+1 / p, \mu \in \mathbb{R}\right\}$.

Обозначим через $\mathfrak{M}_{p}(\Gamma, \omega)$ банахову алгебру операторов, действующих в $L_{p}(\Gamma, \omega)$ и порожденную операторами вида $A=\sum_{k} \prod_{l} A^{k l}$, где $A^{k l}-$ либо СИО $S_{\Gamma}$, либо оператор умножения на функщию $a(x) \in P S V(\Gamma, F)$, и $k, l$ пробегают конечное множество индексов.

Алгебра $\mathfrak{M}_{p}(\Gamma, \omega)$ неприводима и содержит двусторонний идеал компактных операторов $\mathfrak{K}\left(L_{p}(\Gamma, \omega)\right)$. Операторы из алгебры $\mathfrak{M}_{p}(\Gamma, \omega)$ являются операторами локального типа на $\Gamma$.

Положим $\sigma^{x}(A)=\sum_{k} \prod_{l} \sigma^{x}\left(A^{k l}\right)$, где $\sigma^{x}\left(A^{k l}\right)$ определены выше. Отметим, что если $x \in F$, то $\sigma^{x}\left(A^{k l}\right) \in \mathscr{R}_{p, \beta(x)}(N)$, а также что $\sigma^{x}(A)$ в точке $x \in \Gamma \backslash F$ можно отождествить с парой чисел $\left(\sigma_{+}^{x}(A), \sigma_{-}^{x}(A)\right)$.

Пусть $\left\|\sigma^{x}(A)\right\|=\max \left(\left|\sigma_{+}^{x}(A)\right|,\left|\sigma_{-}^{x}(A)\right|\right)$, если $x \in \Gamma \backslash F,\left\|\sigma^{x}(A)\right\|=$ $\left\|\hat{\sigma}^{x}(A)\right\|_{\mathscr{R}_{p, \beta(x)}^{+}(N)}$, если $x \in F$, где $\hat{\sigma}^{x}(A)-$ факторкласс в $\mathscr{R}_{p, \beta(x)}^{+}(N)$, содержащий $\sigma^{x}(A), \sigma(A)=\left\{\sigma^{x}(A)\right\}_{x \in \Gamma}$ и $\|\sigma(A)\|=\sup _{x \in \Gamma}\left\|\sigma^{x}(A)\right\|$. 
Teоpema 1. Пусть $A=\sum_{k} \prod_{l} A^{k l}$. Тогдa

$$
\|\sigma(A)\| \leqslant \inf _{T \in \mathfrak{K}\left(L_{p}(\Gamma, \omega)\right)}\|A-T\|_{\mathfrak{L}\left(L_{p}(\Gamma, \omega)\right)} \leqslant C\|\sigma(A)\| .
$$

Оценка (2) и предельный переход позволяют определить символ $\sigma(A)=$ $\left\{\sigma^{x}(A)\right\}_{x \in \Gamma}$ произвольного оператора $A \in \mathfrak{M}_{p}(\Gamma, \omega)$. В множестве символов операторов из $\mathfrak{M}_{p}(\Gamma, \omega)$ естественным образом вводится структура банаховой алгебры. Эту алгебру мы будем обозначать через $\mathfrak{R}_{p}(\Gamma, \omega)$.

Теорема 2. (а) Отображение $\Sigma_{p}: \mathfrak{M}_{p}(\Gamma, \omega) \rightarrow \mathfrak{N}_{p}(\Gamma, \omega)$, сопоставляющее оператору его символ, есть эпиморфизм банаховой алгебры $\mathfrak{M}_{p}(\Gamma, \omega)$ на банахову алгебру $\mathfrak{N}_{p}(\Gamma, \omega)$, ядром которого является двусторонний идеал $\mathfrak{K}\left(L_{p}(\Gamma, \omega)\right)$.

(b) $A \in \mathfrak{M}_{p}(\Gamma, \omega)$ фредгольмов в $L_{p}(\Gamma, \omega)$ тогда и только тогда, когда его символ обратим в $\mathfrak{N}_{p}(\Gamma, \omega)$, ито равносильно условию: $\sigma_{ \pm}^{x}(A) \neq 0$, когда $x \in \Gamma \backslash F$, и для любой точки $x \in F$ существуют $t_{x} \in \mathbb{R}_{+}$u $C_{x}>0$, такие, что для любой пары $(t, \mu) \in\left(0, t_{x}\right] \times \mathbb{R}$

$$
\left|\operatorname{det} \sigma^{x}(A)(t, \mu+i(-\delta \mu+\beta+1 / p))\right| \geqslant C_{x} .
$$

(с) Если $A \in \mathfrak{M}_{p}(\Gamma, \omega)$ фредгольмов в $L_{p}(\Gamma, \omega)$, то его регуляризатор $R$ также принадлежит алгебре $\mathfrak{M}_{p}(\Gamma, \omega)$ u $\sigma(R)=\sigma(A)^{-1}$.

(d) Индекс фредгольмова оператора $A \in \mathfrak{M}_{p}(\Gamma, \omega)$ вычисляется по формуле

$$
\text { Ind } \begin{aligned}
A=- & \sum_{j=1}^{L} \frac{1}{2 \pi}\left[\arg \frac{\sigma_{+}^{x}(A)}{\sigma_{-}^{x}(A)}\right]_{x \in \gamma_{j}^{\prime}} \\
& \quad-\sum_{j=1}^{M} \frac{1}{2 \pi}\left[\arg \operatorname{det} \sigma^{x_{j}}(A)\left(t_{j}, \mu+i\left(-\delta_{x_{j}} \mu+\beta_{x_{j}}+1 / p\right)\right)\right]_{\mu=-\infty}^{\infty} .
\end{aligned}
$$

В формуле (4) $L$ - число дуг, составляющих контур $\Gamma, M-$ число его узлов $x_{j}$ и $t_{j}$ - произвольные точки из полуинтервалов $\left(0, t_{x_{j}}\right]$, определяемых условием (3). Дуги $\gamma_{j}^{\prime}$ определяются следующим образом. Если кривая $\gamma$ соединяет узлы $x_{j_{1}}$ и $x_{j_{2}}$ и $t_{j_{1}}$ и $t_{j_{2}}-$ зафиксированные выше точки, то $\gamma^{\prime}-$ часть кривой $\gamma$, лежащая между точками $x_{j_{1}}^{\prime}=x_{j_{1}}+\varphi_{j_{1}}\left(t_{j_{1}}\right)$ и $x_{j_{2}}^{\prime}=x_{j_{2}}+\varphi_{j_{2}}\left(t_{j_{2}}\right)$, с ориентацией, индуцированной ориентацией кривой $\gamma$.

\section{ЛитературА}

1. Гохберг И. Ц., Крупник Н. Я. Введение в теорию одномерных сингулярных интегральных операторов. Штиинца, Кишинев, 1973. 2. Roch S., Silberman B. Algebras of convolution operators and their image in the Calkin algebras. Report Math., 9005, Karl Weierstrass Inst. Math., Akad. Wiss. DDR, Berlin, 1990. 3. Симоненко И. Б., Чинь Нгок Минь. Локальный принцип в теории одномерных сингулярных уравнений с кусочно-непрерывными коэффициентами. Ростовский университет, 1986. 4. Bottcher A., Karlovich Yu. I. Integral Equations and Operator Theory, 22, 127-161 (1995). 5. Рабинович В. С. ДАН СССР, 321, №5, 692-696 (1991). 6. Рабинович В. С. Матем. заметки, 58, вып. 1, 65-85 (1995). 